\title{
Quantum spin dynamics: VIII. The master constraint
}

\author{
Thomas Thiemann \\ MPI für Gravitationsphysik, Albert-Einstein-Institut, Am Mühlenberg 1, 14476 Golm near \\ Potsdam, Germany \\ and \\ Perimeter Institute for Theoretical Physics, 31 Caroline Street North, Waterloo, Ontario N2L \\ 2Y5, Canada \\ E-mail: thiemann@aei.mpg.de and tthiemann@perimeterinstitute.ca
}

Received 24 October 2005

Published 14 March 2006

Online at stacks.iop.org/CQG/23/2249

\begin{abstract}
Recently the master constraint programme (MCP) for loop quantum gravity (LQG) was launched which replaces the infinite number of Hamiltonian constraints by a single master constraint. The MCP is designed to overcome the complications associated with the non-Lie-algebra structure of the Dirac algebra of Hamiltonian constraints and was successfully tested in various field theory models. For the case of $3+1$ gravity itself, so far only a positive quadratic form for the master constraint operator was derived. In this paper, we close this gap and prove that the quadratic form is closable and thus stems from a unique self-adjoint master constraint operator. The proof rests on a simple feature of the general pattern according to which Hamiltonian constraints in LQG are constructed and thus extends to arbitrary matter coupling and holds for any metric signature. With this result the existence of a physical Hilbert space for LQG is established by standard spectral analysis.
\end{abstract}

PACS numbers: 04.60.-m, 04.60.Pp

\section{Introduction}

In the canonical approach to quantum gravity one is faced with the task of finding a representation of the Dirac algebra $\mathfrak{D}$ of spatial diffeomorphism and Hamiltonian constraints. This algebra has the following specific features:

(1) The Hamiltonian constraints are not spatially diffeomorphism invariant. In other words, the spatial diffeomorphism constraints form a subalgebra but no ideal of $\mathfrak{D}$.

(2) The Poisson brackets of the Hamiltonian constraints are proportional to a spatial diffeomorphism constraint, however, the coefficients of proportionality are non-trivial functions on phase space, they are 'structure functions' rather than structure constants. In other words, $\mathfrak{D}$ is not a Lie algebra in the strict sense of the word. 
These features follow from very general properties of the hypersurface deformations of a foliation of spacetime and will be ingredients of any canonical approach [1].

In order to quantize the constraints one has to write them in terms of (limits of) elements of a suitable subalgebra $\mathfrak{A}$ of the Poisson algebra of elementary functions on phase space which separates the points. In a second step, one has to look for representations of $\mathfrak{A}$. Loop quantum gravity (LQG) (see [2] for books and [3] for recent reviews) is nothing else than a canonical quantization of general relativity (GR) based on a specific choice of $\mathfrak{A}$, the so-called holonomy-flux algebra. These are functions on phase space which encode the magnetic and electric degrees of freedom of an $S U(2)$ Yang-Mills theory (plus matter) following the real connection formulation of GR due to Ashtekar and Barbero [4]. Such functions are extremely natural from the point of view of (lattice) Yang-Mills theory [5].

Among the multitude of representations of $\mathfrak{A}$ one will be interested in those which are distinguished by physical selection criteria. One such criterion is a unitary representation of the spatial diffeomorphism group (rather than a projective representation thereof). Remarkably, it has been possible to show that such a representation is unique [6]. More precisely, in general, any representation of a $*$-algebra such as $\mathfrak{A}$ is a direct sum of cyclic representations and every cyclic representation comes from a state (positive linear functional) $\omega$ on $\mathfrak{A}$ via the GNS construction [7]. The elements $\varphi$ of the spatial diffeomorphism group act naturally on $\mathfrak{A}$ by automorphisms $a \mapsto \alpha_{\varphi}(a)$ which come from pulling back the magnetic connection one-form and electric field two-form respectively. Hence, it suffices to look for invariant states (the associated representation of the diffeomorphism group is then automatically unitary) and the only such state is the Ashtekar-Isham-Lewandowski (AIL) state [8] $\omega_{0}$ which had been extensively used in LQG even before this uniqueness result transpired.

A particular feature of the AIL representation is the following:

(3) The spatial diffeomorphism group is represented unitarily but not weakly continuously. Hence, by Stone's theorem [9] the infinitesimal self-adjoint generators, i.e. the Lie algebra of spatial diffeomorphisms, does not exist in this representation.

Let us draw some simple conclusions from the results (1)-(3):

(A) Since the infinitesimal generators of spatial diffeomorphisms appear in the classical algebra $\mathfrak{D}$ the representation $\omega_{0}$ of $\mathfrak{A}$ appears to be unsuitable to represent $\mathfrak{D}$.

(B) A way out would be to exponentiate the spatial diffeomorphism and Hamiltonian constraints ('Weyl elements') and to deal with the associated groups they generate. However, while this is possible for the spatial diffeomorphism constraint (by construction of $\omega_{0}$ ), due to (2) there is no (Lie) group structure associated with the Hamiltonian constraints.

(C) Another solution would be to solve first the spatial diffeomorphism constraint. Hence one would extract from the representation space $\mathcal{H}_{0}$ associated with $\omega_{0}$ the (generalized) spatially diffeomorphism invariant elements and assemble them into a Hilbert space $\mathcal{H}_{\text {diff }}$. Then the commutator between two Hamiltonian constraints would be trivial and obstacle (3) would be circumvented. While it is possible to construct $\mathcal{H}_{\text {diff }}$ [10], due to (1) $\mathcal{H}_{\text {diff }}$ does not carry a representation of the Hamiltonian constraints (they do not leave $\mathcal{H}_{\text {diff }}$ invariant).

Thus, (1)-(3) seem to indicate that the structure of LQG must be changed: the representations of the algebras $\mathfrak{D}, \mathfrak{A}$ do not seem to be compatible. However, that is not necessarily the case: in [11-18] the Hamiltonian constraints were quantized on $\mathcal{H}_{0}$ and their commutator indeed annihilates spatially diffeomorphism invariant states (they are generalized zero eigenvectors). This is made possible because the right-hand side of the Poisson bracket between two 
Hamiltonian constraints is not a spatial diffeomorphism constraint. It also involves the structure functions mentioned above and in quantum theory the associated composite operator (product of structure function and spatial diffeomorphism constraint) is less singular than the generator of spatial diffeomorphisms.

Yet, to answer the question whether the algebra of Hamiltonian constraints is properly implemented on $\mathcal{H}_{0}$ is currently unanswered. This is due to the fact that the Hamiltonian constraint is not a polynomial function of the elementary variables and in order to mirror the classical Poisson bracket computation one has to have sufficient control over the spectrum of the volume operator [19, 20]. Work on this, using semiclassical techniques [21], is in progress. However, even after having resolved this issue, due to (2) so far it was not possible to find a constructive procedure to equip the physical states (generalized zero eigenvectors of the Hamiltonian constraint) with an inner product because group averaging techniques (see e.g. [10] and references therein) cannot cope with constraint algebras with structure functions. It would therefore be more desirable to remove the tension between $\mathfrak{A}, \mathfrak{D}$ from the outset and to replace one of them by a classically equivalent algebra such that there are common, manifest representations of both and such that the physical Hilbert space can be constructed.

In [22] the master constraint programme was launched which proposes replacing $\mathfrak{D}$ by a much simpler master constraint algebra $\mathfrak{M}$. Basically, the infinite number of Hamiltonian constraints are replaced by a single constraint, namely the weighted integral of their squares such that the associated master constraint $\mathbf{M}$ is spatially diffeomorphism invariant. For this algebra, spatial diffeomorphisms form an ideal and the commutator of $\mathbf{M}$ with itself is trivial. One can show that $\mathfrak{D}, \mathfrak{M}$ are classically equivalent. The physical Hilbert is then readily available using standard spectral analysis techniques [22, 23] provided one manages to implement $\mathbf{M}$ as a self-adjoint operator $\widehat{\mathbf{M}}$ on either $\mathcal{H}_{0}$ or $\mathcal{H}_{\text {diff }}$ (and provided that the Hilbert space is a direct sum of separable subspaces invariant for $\widehat{\mathbf{M}}$ ). To take the sum of squares of constraints rather than the constraints themselves has successfully been tested for various toy models including those with an infinite number of degrees of freedom and with structure functions [24].

In [22] we proposed a quadratic form $Q_{\mathbf{M}}$ for the master constraint on a dense domain of $\mathcal{H}_{\text {diff }}\left(Q_{\mathbf{M}}\right.$ is a graph changing, diffeomorphism invariant quadratic form and cannot exist on $\mathcal{H}_{0}$; see [22] for details). We also constructed a quadratic form $Q_{\mathbf{M}_{E}}$ for the extended master constraint on $\mathcal{H}_{0}$ which also involves the weighted integral of the square of the spatial diffeomorphism constraint (possible because $Q_{\mathbf{M}_{E}}$ is not graph changing). Two issues were left open in that paper:

(1) A systematic derivation of $Q_{\mathrm{M}}$ was not given.

(2) It was not demonstrated that $Q_{\mathrm{M}}$ is closable and is the quadratic form of a unique selfadjoint operator $\widehat{\mathbf{M}}$. The same applies to $Q_{\mathbf{M}_{E}}$.

In this paper we close this gap.

In section 2, we derive $Q_{\mathrm{M}}$ using the known regularization of the Hamiltonian constraint.

This is technically non-trivial because the Hamiltonian constraint can only be defined on $\mathcal{H}_{0}$ while $Q_{\mathrm{M}}$ can only be defined on $\mathcal{H}_{\text {diff }}$. The new technical tool necessary for the derivation is the extension of the scalar product on $\mathcal{H}_{\text {diff }}$ to all elements of the algebraic dual Cyl ${ }^{*}$ of the space Cyl of finite linear combinations of spin network functions.

In section 3, we prove that $Q_{\mathrm{M}}$ is closable. We also show that the proof extends to all matter coupling [16] and to $Q_{\mathbf{M}_{E}}$.

In section 4 , we display explicitly a separable subspace of $\mathcal{H}_{\text {diff }}$ which is left invariant by $\widehat{\mathbf{M}}$ and which should capture the full physics of LQG. 
In section 5, we show that the master equation, which is a condition on weak Dirac observables, is well defined without supplementing the master constraint with boundary terms in the presence of asymptotically flat boundary conditions.

In the same section, we conclude and outline the further steps in the task to solve the quantum dynamics of LQG.

\section{Derivation of the quadratic form of the master constraint}

The derivation of the quadratic form of the master constraint will be given only for the full Lorentzian Hamiltonian constraint for pure gravity. We will show then that the same derivation applies to all matter couplings with just more terms to write.

\subsection{Strategy}

The strategy to implement the master constraint is as follows. Let $\mathcal{T}(\epsilon)$ be a triangulation of $\sigma$, e.g. into tetrahedra $\Delta$, and denote by $\epsilon \rightarrow 0$ any limit in which the triangulation is infinitely refined subject to the constraints on a refinement that one uses in defining Riemannian integrals.

Recall $[11,12]$ that up to a constant the Lorentzian Hamiltonian constraint of pure gravity is given by

$$
\begin{aligned}
& H(N)=\int_{\sigma} \mathrm{d}^{3} x N(x) H(x)=a H_{E}(N)+b K(N) \\
& H_{E}(N)=\int_{\sigma} \mathrm{d}^{3} x N(x) H_{E}(x)=\int_{\sigma} N(x) \operatorname{Tr}\left(F(x) \wedge\left\{A(x), V\left(R_{x}\right)\right\}\right) \\
& K(N)=\int_{\sigma} \operatorname{Tr}\left(\left\{A(x),\left\{H_{E}(1), V(\sigma)\right\}\right\} \wedge\left\{A(x),\left\{H_{E}(1), V(\sigma)\right\}\right\} \wedge\left\{A(x), V\left(R_{x}\right)\right\}\right)
\end{aligned}
$$

where $x \mapsto H(x)$ denotes the Hamiltonian constraint and $\sigma$ is a three-manifold such that $\mathbb{R} \times \sigma$ is diffeomorphic to the spacetime manifold $M$. Here $A$ is the gravitational $S U(2)$ connection, $F$ its curvature, $N$ the lapse function, $R_{x}$ is any open region containing $x$ and

$$
V(R):=\int_{R} \mathrm{~d}^{3} x \sqrt{|\operatorname{det}(E)|}(x)
$$

is the volume of $R$ with $E$ the electric field vector density. The non-vanishing canonical brackets are $\left\{E_{j}^{a}(x), A_{b}^{k}(y)\right\}=\kappa \beta \delta_{b}^{a} \delta_{j}^{k} \delta(x, y)$ where $\kappa=8 \pi G, G$ is Newton's constant and $\beta$ is the Immirzi parameter [25]. The real constants $a, b$ in (2.1) also depend on $\beta$.

The integral (2.1) is the limit of the Riemannian sum

$$
H(N)=\lim _{\epsilon \rightarrow 0} \sum_{\Delta \in \mathcal{T}(\epsilon)} N(v(\Delta)) H(\Delta)
$$

where $v(\Delta)$ is an interior point of $\Delta$ and $H(\Delta)=H\left(\chi_{\Delta}\right)$ where $\chi_{\Delta}$ is the characteristic function of the set $\Delta$. That is, $H(\Delta)=H(N)_{N=\chi_{\Delta}}$.

Then the classical master constraint as defined in [22]

$$
\mathbf{M}=\int_{\sigma} \mathrm{d}^{3} x \frac{[H(x)]^{2}}{\sqrt{\operatorname{det}(q)}(x)}
$$

is likewise the limit of the Riemannian sum

$$
\mathbf{M}=\lim _{\epsilon \rightarrow 0} \sum_{\Delta \in \mathcal{T}(\epsilon)} \frac{H(\Delta)^{2}}{V(\Delta)}
$$

where $H(\Delta)=H\left(\chi_{\Delta}\right), V(\Delta)=\int_{\Delta} \mathrm{d}^{3} x \sqrt{|\operatorname{det}(E)|}$ as above. 
We now choose w.l.g. the $R_{x}, x \in \Delta$ to actually coincide with $\Delta$ (only $x \in \partial \Delta$ are not interior points of $\Delta$ but these form a set of measure zero). Then

$C(\Delta):=\frac{H(\Delta)}{\sqrt{V(\Delta)}}=\int_{\Delta} \operatorname{Tr}\left(F \wedge \frac{\{A, V(\Delta)\}}{\sqrt{V(\Delta)}}\right)=2 \int_{\Delta} \operatorname{Tr}(F \wedge\{A, \sqrt{V(\Delta)}\})$

where we used $\{., V(\Delta)\} / \sqrt{V(\Delta)}=2\{., \sqrt{V(\Delta)}\}$ and thus

$$
\mathbf{M}=\lim _{\epsilon \rightarrow 0} \sum_{\Delta \in \mathcal{T}(\epsilon)} C(\Delta)^{2} .
$$

Note that $C(\Delta)$ is up to a factor of 2 the same as $H(\Delta)$, just that $V(\Delta)$ is replaced by $\sqrt{V(\Delta)}$. This is convenient because the $C(\Delta)$ can then be quantized precisely as the $H(\Delta)$ in $[12,16]$ with this simple change in the power of the volume operator. All the qualitative features remain the same, only the numerical values of the matrix elements of the corresponding regularized operators $\hat{C}_{\epsilon}^{\dagger}(\Delta)$ change. Note that in contrast to $\hat{H}(\Delta)^{\dagger}$ the operator $\hat{C}_{\epsilon}^{\dagger}(\Delta)$ depends in addition to the smearing function $\chi_{\Delta}$ on $\epsilon$ because we have to use $\sqrt{V(\Delta)}$ while for $\hat{H}(\Delta)^{\dagger}$ we may use $V(\sigma)$.

We denote the quantization of $C(\Delta)$, densely defined on the finite linear span $\mathcal{D}$ of spin network functions (which constitute a basis of $\mathcal{H}_{0}$ ), by $\hat{C}_{\epsilon}^{\dagger}(\Delta)$ rather than $\hat{C}_{\epsilon}(\Delta)$ because of the definition of the dual operator $\hat{C}_{\epsilon}^{\prime}(\Delta)$ on the algebraic dual $\mathcal{D}^{*}$ (linear functionals on $\mathcal{D}$ without continuity assumptions): for $l \in \mathcal{D}^{*}$ and $f \in \mathcal{D}$ we have $\left[\hat{C}_{\epsilon}^{\prime}(\Delta) l\right](f):=l\left(\hat{C}_{\epsilon}^{\dagger}(\Delta) f\right)$. Classically $C(\Delta)$ is real valued so that $\hat{C}_{\epsilon}^{\dagger}(\Delta)$ qualifies as a quantization of $C(\Delta)=\overline{C(\Delta)}$. Note, however, that $\hat{C}_{\epsilon}^{\dagger}(\Delta)$ must not be symmetric for reasons of the absence of anomalies in the constraint algebra; see e.g. [23] and references therein.

One may therefore be tempted to simply compute the regularized dual operators $\hat{C}_{\epsilon}^{\prime}(\Delta)$ on $\mathcal{D}^{*}$ and then to restrict it to $\mathcal{D}_{\text {diff }}^{*}$ (the spatially diffeomorphism invariant elements of $\mathcal{D}^{*}$ [10]). Using the fact that $C(\Delta)$ is real valued we may write $(2.7)$ as

$$
\mathbf{M}=\lim _{\epsilon \rightarrow 0} \sum_{\Delta \in \mathcal{T}(\epsilon)} \overline{C(\Delta)} C(\Delta)
$$

and since we must implement $\widehat{\mathbf{M}}$ directly on $\mathcal{H}_{\text {Diff }}$ (the Hilbert space completion of the finite linear span of spatially diffeomorphism group averaged spin networks functions [10]) one would like to try to define the quadratic form

$$
Q_{\mathbf{M}}\left(l, l^{\prime}\right):=\lim _{\epsilon \rightarrow 0} \sum_{\Delta \in \mathcal{T}(\epsilon)}\left\langle l,\left(\hat{C}_{\epsilon}^{\prime}(\Delta)\right)^{*} \hat{C}_{\epsilon}^{\prime}(\Delta) l^{\prime}\right\rangle_{\mathrm{diff}}=\lim _{\epsilon \rightarrow 0} \sum_{\Delta \in \mathcal{T}(\epsilon)}\left\langle\hat{C}_{\epsilon}^{\prime}(\Delta) l, \hat{C}_{\epsilon}^{\prime}(\Delta) l^{\prime}\right\rangle_{\mathrm{diff}}
$$

where (.) $)^{*}$ denotes the adjoint operation on $\mathcal{H}_{\text {diff }}$. However, at least at finite $\epsilon$ equation (2.9) is ill defined because we are using the scalar product on $\mathcal{H}_{\text {diff }}$ while $\hat{C}_{\epsilon}^{\prime}(\Delta) l \notin \mathcal{H}_{\text {diff. }}$. In other words, just as the Hamiltonian constraint, $C(\Delta)$ is not spatially diffeomorphism invariant and $C_{\epsilon}^{\prime}(\Delta)$ does not preserve $\mathcal{H}_{\text {diff }}$. For the same reason the adjoint operation with respect to $\mathcal{H}_{\text {diff }}$ carried out in the second step is unjustified.

\subsection{New inner product on algebraic dual}

The hope is, of course, that (2.9) makes sense in the limit $\epsilon \rightarrow 0$ when the corresponding classical quantity becomes spatially diffeomorphism invariant. The new tool to arrive at this and which we introduce here for the first time in LQG is to equip the space $\mathcal{D}^{*}$ with an inner product which reduces to that on $\mathcal{H}_{\text {Diff }}$ when evaluated on $\mathcal{D}_{\text {diff }}^{*}$.

We will now, formally, define this inner product and start with some preparations. By $\mathcal{S}$ we denote the space of labels of spin network functions and we write $s$ for its elements and $T_{s}$ for spin network functions. The orbit under (semianalytic [6]) diffeomorphisms is 
given by $[s]:=\{\varphi \cdot s ; \varphi \in \operatorname{Diff}(\sigma)\}$ where $s \mapsto \varphi \cdot s$ denotes the action of diffeomorphisms on spin network labels. Basically a spin network label $s$ is a triple $s=(\gamma(s), j(s), I(s))$ consisting of a semianalytic graph $\gamma$, a labelling $j$ of its edges with non-vanishing spin quantum numbers $j$ and a labelling of its vertices with gauge invariant intertwiners $I$. Then $\varphi \cdot s=(\varphi(\gamma(s)), j(s), I(s))$. Given a spin network diffeomorphism equivalence class $[s]$ we define the non-standard number or Cantor aleph

$$
\aleph([s]):=|[s]|:=\left|\left\{s^{\prime} \in \mathcal{S} ;\left[s^{\prime}\right]=[s]\right\}\right|
$$

as the size of the orbit $[s]$. Now recall [10] that preferred elements of $\mathcal{D}_{\text {diff }}^{*}$ were given by

$$
l_{[s]}:=\sum_{s^{\prime} \in[s]}\left\langle T_{s^{\prime}}, .\right\rangle_{\mathrm{kin}}, \quad \eta\left(T_{s}\right)=\eta_{[s]} l_{[s]}
$$

with positive numbers $\eta_{[s]}$ and

$$
\left\langle\eta\left(T_{s}\right), \eta\left(T_{s^{\prime}}\right)\right\rangle_{\text {diff }}=\eta\left(T_{s^{\prime}}\right)\left[T_{s}\right] .
$$

Here $\eta$ denotes the group averaging or rigging map introduced in [10] and $\langle., .\rangle_{\text {kin }}$ denotes the inner product on $\mathcal{H}_{0}$.

An arbitrary element of $\mathcal{D}^{*}$ is of the form $l=\sum_{s \in \mathcal{S}} c_{s}\left\langle T_{s}, .\right\rangle_{\text {kin }}$. Formally, we may define an inner product $\langle.,\rangle_{*}$ on $\mathcal{D}^{*}$ by

$$
\begin{aligned}
\left\langle l, l^{\prime}\right\rangle_{*} & :=\sum_{s, s^{\prime}} \overline{c_{s}} c_{s^{\prime}}^{\prime}\left\langle\left\langle T_{s}, .\right\rangle_{\mathrm{kin}},\left\langle T_{s^{\prime}}, .\right\rangle_{\mathrm{kin}}\right\rangle_{*} \\
& :=\sum_{s, s^{\prime}} \overline{c_{s}} c_{s^{\prime}}^{\prime}\left\langle T_{s^{\prime}}, T_{s}\right\rangle_{\mathrm{kin}} \frac{\sqrt{\eta_{[s]} \eta_{\left[s^{\prime}\right]}}}{\sqrt{\aleph([s]) \aleph\left(\left[s^{\prime}\right]\right)}}=\sum_{s} \overline{c_{s}} c_{s}^{\prime} \frac{\eta_{[s]}}{\aleph([s])} .
\end{aligned}
$$

This reproduces the inner product between the $\eta_{[s]}$ which correspond to $c_{s^{\prime}}=\chi_{[s]}\left(s^{\prime}\right)$. It also formally corresponds to formally extending (2.13) to $\mathcal{H}_{\text {kin }}$ with

$$
\left\langle T_{s}, T_{s^{\prime}}\right\rangle_{*}:=\left\langle T_{s}, T_{s^{\prime}}\right\rangle_{\mathrm{kin}} \frac{\sqrt{\eta_{[s]} \eta_{\left[s^{\prime}\right]}}}{\sqrt{\aleph([s]) \aleph\left(\left[s^{\prime}\right]\right)}}
$$

but of course elements of $\mathcal{H}_{\text {Kin }}$ have zero norm in this inner product. Hence by far not all elements of $\mathcal{D}^{*}$ are normalizable in this inner product and many elements have zero norm with respect to it. By passing to the quotient by the null vectors and completing we may turn the normalizable elements of $\mathcal{D}^{*}$ into a Hilbert space $\mathcal{H}_{*} \subset \mathcal{D}^{*}$. Note that (2.13) is the first inner product to be proposed on (a subset of) $\mathcal{D}^{*}$.

It is curious to note that we may formally define a partial isometry

$$
V: \mathcal{H}_{*} \rightarrow \mathcal{H}_{\text {Kin }}, \quad l=\sum_{s} c_{s}\left\langle T_{s}, .\right\rangle_{\mathrm{Kin}} \mapsto \tilde{l}=\sum_{s} c_{s} \sqrt{\frac{\eta_{[s]}}{\kappa([s])}} T_{s}
$$

so that we may formally identify $\langle., .\rangle_{*}$ with the kinematical inner product $\langle., .\rangle_{\text {kin }}$ under the $\operatorname{map} l \mapsto \tilde{l}$.

In our application of $\langle., .\rangle_{*}$ quotients of non-standard numbers will appear and this is a subtle issue in general [26]. Fortunately, the quotients we will find all equal unity or zero by inspection.

\subsection{Derivation of the quadratic form}

The idea is then to use $\langle., .\rangle_{*}$ and its associated adjoint operation to define (2.9) properly, that is,

$$
Q_{\mathbf{M}}\left(l, l^{\prime}\right):=\lim _{\epsilon \rightarrow 0} \sum_{\Delta \in \mathcal{T}(\epsilon)}\left\langle l,\left(\hat{C}_{\epsilon}^{\prime}(\Delta)\right)^{*} \hat{C}_{\epsilon}^{\prime}(\Delta) l^{\prime}\right\rangle_{*}=\lim _{\epsilon \rightarrow 0} \sum_{\Delta \in \mathcal{T}(\epsilon)}\left\langle\hat{C}_{\epsilon}^{\prime}(\Delta) l, \hat{C}_{\epsilon}^{\prime}(\Delta) l^{\prime}\right\rangle_{*}
$$


where (.)* is now the adjoint operation on $\mathcal{H}_{*}$ and (2.16) is now well defined. To evaluate $\langle., .\rangle_{*}$ we write

$$
\hat{C}_{\epsilon}^{\prime}(\Delta) l=\sum_{s \in \mathcal{S}} c_{s}^{l}(\Delta, \epsilon)\left\langle T_{s}, .\right\rangle_{\mathrm{kin}} \quad \Rightarrow \quad c_{s}^{l}(\Delta, \epsilon)=l\left(C_{\epsilon}^{\dagger}(\Delta) T_{s}\right) .
$$

Hence (2.17) becomes

$$
\begin{aligned}
Q_{\mathbf{M}}\left(l, l^{\prime}\right) & =\lim _{\epsilon \rightarrow 0} \sum_{\Delta \in \mathcal{T}(\epsilon)} \sum_{s} \overline{c_{s}^{l}(\Delta, \epsilon)} c_{s}^{l^{\prime}}(\Delta, \epsilon) \frac{\eta_{[s]}}{\aleph([s])} \\
& =\lim _{\epsilon \rightarrow 0} \sum_{\Delta \in \mathcal{T}(\epsilon)} \sum_{[s]} \frac{\eta_{[s]}}{\aleph([s])} \sum_{s^{\prime} \in[s]} \overline{c_{s^{\prime}}^{l}(\Delta, \epsilon)} c_{s^{\prime}}^{l^{\prime}}(\Delta, \epsilon) .
\end{aligned}
$$

We note that for given $l, l^{\prime}$ only a finite number of $[s]$ contribute to (2.18): namely, both $l, l^{\prime}$ are finite linear combinations of the $l_{\left[s_{1}\right]}$ in (2.11), hence it suffices to show that for any $\left[s_{1}\right],\left[s_{2}\right]$ the numbers

$$
\overline{c_{s^{\prime}}^{l_{\left[s_{1}\right.}}(\Delta, \epsilon)} c_{s^{\prime}}^{l_{\left.s_{2}\right]}}(\Delta, \epsilon)
$$

are non-vanishing only when $s^{\prime} \in[s]$ and $[s]$ ranges over a finite number of classes. In order that $c_{s^{\prime}}^{l_{\left[s_{1}\right]}}(\Delta, \epsilon) \neq 0$ we must have that $\hat{C}_{\epsilon}^{\dagger}(\Delta) T_{s^{\prime}}$ is a finite linear combination of spin network states which involves at least one of the $T_{s_{1}^{\prime}}$ with $s_{1}^{\prime} \in\left[s_{1}\right]$. But from the explicit action of $\hat{C}_{\epsilon}^{\dagger}(\Delta)$ [12] it is clear that for each $s_{1}^{\prime} \in\left[s_{1}\right]$ there is only a finite set $\mathcal{S}\left(s_{1}^{\prime}\right)$ of $s^{\prime}$ with this property ${ }^{1}$. Moreover, for each $s_{1}^{\prime} \in\left[s_{1}\right]$ the number of elements of $\mathcal{S}\left(s_{1}^{\prime}\right)$ is the same and the classes of the elements of $\mathcal{S}\left(s_{1}^{\prime}\right)$ do not depend on the representative $s_{1}^{\prime} \in\left[s_{1}\right]$. Denote the finite set of these classes by $[\mathcal{S}]\left(\left[s_{1}\right]\right)$.

The sum over $[s]$ in $(2.18)$ is therefore only over the finite set $[\mathcal{S}]\left(\left[s_{1}\right]\right) \cap[\mathcal{S}]\left(\left[s_{2}\right]\right)$ for $l=l_{\left[s_{1}\right]}, l^{\prime}=l_{\left[s_{2}\right]}$, hence for any $l, l^{\prime} \in \mathcal{D}_{\text {diff }} \subset \mathcal{H}_{\text {Diff }}$ the sum over $[s]$ in (2.18) is finite ${ }^{2}$. We may therefore interchange the sum $\sum_{[s]}$ with the $\sum_{\Delta}$ and the $\operatorname{limit}_{\lim } \rightarrow 0$ and arrive at

$$
Q_{\mathbf{M}}\left(l, l^{\prime}\right)=\sum_{[s]} \frac{\eta_{[s]}}{\aleph([s])} \lim _{\epsilon \rightarrow 0} \sum_{\Delta \in \mathcal{T}(\epsilon)} \sum_{s^{\prime} \in[s]} \overline{c_{s^{\prime}}^{l}(\Delta, \epsilon)} c_{s^{\prime}}^{l^{\prime}}(\Delta, \epsilon) .
$$

Fix $s^{\prime} \in[s]$ and consider $\hat{C}_{\epsilon}^{\dagger}(\Delta) T_{s^{\prime}}$. From [12] we know that this can be written in the form

$$
\hat{C}_{\epsilon}^{\dagger}(\Delta) T_{s^{\prime}}=\sum_{v \in V\left(\gamma\left(s^{\prime}\right)\right) \cap \Delta} \hat{C}_{\epsilon, \delta, \gamma\left(s^{\prime}\right), v}^{\dagger} T_{s^{\prime}}
$$

where the operators $\hat{C}_{\epsilon, \delta \gamma\left(s^{\prime}\right), v}^{\dagger}$ involve only degrees of freedom associated with edges of $\gamma\left(s^{\prime}\right)$ in the vicinity of $v$ and additional loops attached to a neighbourhood of $v$ which have to be chosen within the diffeomorphism invariance class specified in [12] and whose choice has been denoted by a choice function $\delta$.

For sufficiently small $\epsilon$ each $\Delta$ contains at most one vertex and the sum over $\Delta$ therefore reduces to the finite set $\mathcal{T}\left(\epsilon, s^{\prime}\right)$ of those $\Delta$ containing precisely one vertex of $\gamma\left(s^{\prime}\right)$. We may therefore interchange the sum $\sum_{s^{\prime}}$ with the $\sum_{\Delta}$ and the limit $\epsilon \rightarrow 0$ and obtain

$$
\begin{aligned}
Q_{\mathbf{M}}\left(l, l^{\prime}\right) & =\sum_{[s]} \frac{\eta_{[s]}}{\aleph([s])} \sum_{s^{\prime} \in[s]} \lim _{\epsilon \rightarrow 0} \sum_{\Delta \in \mathcal{T}\left(\epsilon, s^{\prime}\right)} \overline{c_{s^{\prime}}^{l}(\Delta, \epsilon)} c_{s^{\prime}}^{l^{\prime}}(\Delta, \epsilon) \\
& =\sum_{[s]} \frac{\eta_{[s]}}{\aleph([s])} \sum_{s^{\prime} \in[s]} \lim _{\epsilon \rightarrow 0} \sum_{v \in V\left(\gamma\left(s^{\prime}\right)\right.} \overline{c_{s^{\prime}}^{l}(v, \epsilon, \delta)} c_{s^{\prime}}^{l^{\prime}}(v, \epsilon, \delta)
\end{aligned}
$$

\footnotetext{
1 This holds only when we restrict the Hilbert space $\mathcal{H}_{\text {diff }}$ (and correspondingly the vector space $\mathcal{D}^{*}$ ) to one of the invariant and separable Hilbert spaces defined in section 4. See also [22].

${ }^{2} \mathcal{D}_{\text {diff }}$ is the dense subset of $\mathcal{H}_{\text {diff }}$ consisting of the finite linear span of the $l_{[s]}$. Both are subspaces of $\mathcal{D}_{\text {diff }}^{*}$.
} 


$$
\begin{aligned}
& =\sum_{[s]} \frac{\eta_{[s]}}{\aleph([s])} \sum_{s^{\prime} \in[s]} \sum_{v \in V\left(\gamma\left(s^{\prime}\right)\right)} \overline{c_{s^{\prime}}^{l}(v, \delta)} c_{s^{\prime}}^{l^{\prime}}(v, \delta) \\
& =\sum_{[s]} \frac{\eta_{[s]}}{\aleph([s])} \sum_{s^{\prime} \in[s]} \sum_{v \in V\left(\gamma\left(s^{\prime}\right)\right)} \overline{c_{s^{\prime}}^{l}(v)} c_{s^{\prime}}^{l^{\prime}}(v)
\end{aligned}
$$

where

$$
c_{s^{\prime}}^{l}(v, \epsilon, \delta)=l\left(\hat{C}_{\epsilon, v, \delta}^{\dagger} T_{s^{\prime}}\right)=l\left(\hat{C}_{v, \delta}^{\dagger} T_{s^{\prime}}\right)=l\left(\hat{C}_{v, \delta_{0}}^{\dagger} T_{s^{\prime}}\right)=: c_{s^{\prime}}^{l}(v) .
$$

Here in the second equality of (2.23) the $\epsilon$ dependence coming from $V(\delta)$ has dropped out since $\Delta$ is so small that it contains only $v \in V\left(\gamma\left(s^{\prime}\right)\right)$ and in the third equality we could fix $\delta=\delta_{0}$ by spatial diffeomorphism invariance of $l$. In the second step in (2.22) the sum over the contributing $\Delta$ could be replaced by the sum over vertices and since then nothing depends on $\epsilon$ any more the limit $\epsilon \rightarrow 0$ is trivial.

We now claim that

$$
a\left(s^{\prime}\right):=\sum_{v \in V\left(\gamma\left(s^{\prime}\right)\right)} \overline{c_{s^{\prime}}^{l}(v)} c_{s^{\prime}}^{l^{\prime}}(v)
$$

only depends on the class $[s]$ of $s^{\prime}$. Indeed,

$$
\begin{aligned}
a\left(\varphi \cdot s^{\prime}\right) & =\sum_{v \in V\left(\gamma\left(\varphi \cdot s^{\prime}\right)\right)} \overline{c_{\varphi \cdot s^{\prime}}^{l}(v)} c_{\varphi \cdot s^{\prime}}^{l^{\prime}}(v) \\
& =\sum_{v \in \varphi\left(V\left(\gamma\left(s^{\prime}\right)\right)\right)} \overline{c_{\varphi \cdot s^{\prime}}^{l}(v)} c_{\varphi \cdot s^{\prime}}^{l^{\prime}}(v) \\
& =\sum_{v \in V\left(\gamma\left(s^{\prime}\right)\right)} \overline{c_{\varphi \cdot s^{\prime}}^{l}(\varphi(v))} c_{\varphi \cdot s^{\prime}}^{l^{\prime}}(\varphi(v))
\end{aligned}
$$

but

$$
\begin{aligned}
c_{\varphi \cdot s^{\prime}}^{l}(\varphi(v)) & =l\left(\hat{C}_{\varphi(v), \delta_{0}}^{\dagger} \hat{U}(\varphi) T_{s^{\prime}}\right)=l\left(\hat{U}(\varphi) \hat{C}_{v, \delta_{0}^{\prime}}^{\dagger} T_{s^{\prime}}\right) \\
& =l\left(\hat{C}_{v, \delta_{0}}^{\dagger} T_{s^{\prime}}\right)=l\left(\hat{C}_{v, \delta_{0}}^{\dagger} T_{s^{\prime}}\right) \\
& =c_{s^{\prime}}^{l}(v)
\end{aligned}
$$

where in the first step we used that $\operatorname{Diff}_{s a}^{\omega}(\sigma)$ is unitarily implemented [10], in the second we have used the covariance relation up to a diffeomorphism $\hat{U}(\varphi) \hat{H}_{\delta_{0}}(N) \hat{U}(\varphi)^{-1}=\hat{H}_{\delta_{0}^{\prime}}\left(\varphi^{*} N\right)$ established in [12] under which the choice $\delta_{0}$ may change to some $\delta_{0}^{\prime}$ but two choices are related by a diffeomorphism [12] and in the last two steps we used diffeomorphism invariance of $l$.

It follows that all the $\mathcal{\aleph}([s])$ terms in the sum $\sum_{s^{\prime} \in[s]}$ are identical. Let $s_{0}([s])$ be a representative of $[s]$ then we may finish our derivation and get the final result

$$
Q_{\mathbf{M}}\left(l, l^{\prime}\right)=\sum_{[s]} \eta_{[s]} \sum_{v \in V\left(\gamma\left(s_{0}[s]\right)\right)} \overline{l\left(\hat{C}_{v}^{\dagger} T_{s_{0}([s])}\right)} l^{\prime}\left(\hat{C}_{v}^{\dagger} T_{s_{0}([s])}\right) .
$$

We have dropped the irrelevant label $\delta_{0}$. Since we showed that the sum over $[s]$ collapses to a finite number of terms, (2.27) is well defined.

Readers who dislike the formal steps performed involving division by and summing over $\aleph([s])$ terms may take (2.27) as a definition. Alternatively, one may dive into the field of non-standard analysis [26] and regularize the sums over uncountably infinite number of terms. 


\section{The master constraint operator}

Having constructed a quadratic form densely defined on the dense subspace $\mathcal{D}_{\text {diff }} \subset \mathcal{D}_{\text {diff }}^{*}$ of $\mathcal{H}_{\text {diff }}$ given by the finite linear span of elements of the form $l_{[s]}$ we want to show that $Q_{\mathrm{M}}$ is associated with a unique self-adjoint operator $\widehat{\mathbf{M}}$. This is not trivial as the following reveals [9].

\section{Definition 3.1.}

(i) Let $T$ be a densely defined operator on a Hilbert space $\mathcal{H} . \Gamma(T):=\{(l, T l) ; l \in D(T)\} \subset$ $\mathcal{H} \times \mathcal{H}$ is called the graph of $T . T$ is called closed if the set $\Gamma(T)$ is closed with respect to the inner product $\left\langle\left(l_{1}, l_{2}\right),\left(l_{1}^{\prime}, l_{2}^{\prime}\right)\right\rangle=\left\langle l_{1}, l_{1}^{\prime}\right\rangle+\left\langle l_{2}, l_{2}^{\prime}\right\rangle$. T is called closable if it has a closed extension to $D(\bar{T})$, that is, $D(T) \subset D(\bar{T})$ and $\bar{T}_{\mid D(T)}=T$. The smallest closed extension is called the closure $\bar{T}$.

(ii) A quadratic form $Q$ on a Hilbert space $\mathcal{H}$ is a sesqui-linear form on $D(Q) \times D(Q)$ where $D(Q)$ is a dense form domain. A quadratic form is called semibounded provided that $Q(l, l) \geqslant-c\|l\|^{2}$ for some $c \geqslant 0$ and positive if $c=0$. A semibounded quadratic form $Q$ is called closed provided that $D(Q)$ is complete in the norm $\|l\|_{+1}=\sqrt{Q(l, l)+c\|l\|^{2}}$. If $Q$ is closed and $D^{\prime}(Q) \subset D(Q)$ is dense then $D^{\prime}(Q)$ is called a form core.

\section{Theorem 3.1.}

(i) Let $T$ be a symmetric operator $\left(D(T) \subset D\left(T^{*}\right), T_{\mid D(T)}^{*}=T\right)$. Then $T$ is closable, however, its closure may not be self-adjoint $\left(D(\bar{T}) \neq D\left(\bar{T}^{\dagger}\right)\right.$.

(ii) Let $Q$ be a semi-bounded quadratic form. Then $Q$ may not be closable, but if it is and the closure is semi-bounded, then $Q$ is the quadratic form of a unique self-adjoint operator $T$ according to $Q\left(l, l^{\prime}\right)=\left\langle l, T l^{\prime}\right\rangle=: Q_{T}\left(l, l^{\prime}\right)$.

(iii) Let $T$ be a positive, symmetric operator. Then the corresponding positive quadratic form $Q_{T}$ has a positive closure $\overline{Q_{T}}$. The unique positive operator $\tilde{T}$ corresponding to that closure via $Q_{\tilde{T}}=\overline{Q_{T}}$ is called the Friedrichs extension of T. It may extend the closure $\bar{T}$ of $T$ and is the only self-adjoint extension which contains $D\left(\overline{Q_{T}}\right)$.

In our case, we can take as $D\left(Q_{\mathbf{M}}\right):=\mathcal{D}_{\text {diff }}$ the finite linear span of the $l_{[s]}$. Our $Q_{\mathbf{M}}$ is manifestly positive and sesqui-linear. It remains to show that it is closable. The problem that one might encounter is the following: the Hilbert space $\mathcal{H}_{\text {diff }}$ has the orthonormal basis $T_{[s]}:=l_{[s]} / \sqrt{\eta_{[s]}}$ and we would like to define an operator $\widehat{\mathbf{M}}$ densely on $D\left(Q_{\mathbf{M}}\right)$ by

$$
\widehat{\mathbf{M}} T_{\left[s_{2}\right]}:=\sum_{\left[s_{1}\right]} Q_{\mathbf{M}}\left(T_{\left[s_{1}\right]}, T_{[s]}\right) T_{\left[s_{1}\right]} .
$$

However, the right-hand side should be an element of $\mathcal{H}_{\text {diff }}$, that is

$$
\left\|\widehat{\mathbf{M}} T_{\left[s_{2}\right]}\right\|^{2}:=\sum_{\left[s_{1}\right]}\left|Q_{\mathbf{M}}\left(T_{\left[s_{1}\right]}, T_{\left[s_{2}\right]}\right)\right|^{2}<\infty .
$$

Hence there is a convergence issue to be resolved.

\section{Theorem 3.2.}

(i) The positive quadratic form $Q_{\mathbf{M}}(2.27)$ is closable and induces a unique, positive selfadjoint operator $\widehat{\mathbf{M}}$ on $\mathcal{H}_{\text {diff }}$.

(ii) Moreover, the point zero is contained in the point spectrum of $\widehat{\mathbf{M}}$. 
Proof of theorem 3.2. (i) Since, given $\left[s_{2}\right]$ the 'matrix element' $Q_{\mathbf{M}}\left(T_{\left[s_{1}\right]}, T_{\left[s_{2}\right]}\right)$ is finite for every $\left[s_{1}\right],\left[s_{2}\right]$ in order to prove convergence of (3.2) it will be sufficient to show that $Q_{\mathrm{M}}\left(T_{\left[s_{1}\right]}, T_{\left[s_{2}\right]}\right) \neq 0$ for at most a finite number of $\left[s_{1}\right]$ only.

(1) Let us fix $\left[s_{1}\right],\left[s_{2}\right]$ and consider the term corresponding to $[s]$ in (2.27). In order that it does not vanish the expression

$$
\sum_{v \in V\left(\gamma\left(s_{0}[s]\right)\right)} \overline{T_{\left[s_{1}\right]}\left(\hat{C}_{v}^{\dagger} T_{s_{0}([s])}\right)} T_{\left[s_{2}\right]}\left(\hat{C}_{v}^{\dagger} T_{s_{0}([s])}\right)
$$

must be nonzero. Hence the spin network decomposition of $\hat{C}_{v}^{\dagger} T_{s_{0}([s])}$ must contain a term diffeomorphic to $T_{s_{1}}$ and a term diffeomorphic to $T_{s_{2}}$ for at least one $v \in V\left(\gamma\left(s_{0}([s])\right)\right)$. Let us estimate the number of $[s]$ for which this is possible. The action of $\hat{C}_{v}^{\dagger}$ on $T_{s_{0}([s])}$ consists of two terms, corresponding to $H_{E}(N)$ and $K(N)$ in (2.1) respectively (with the changed power of the volume operator):

First term. The first term adds an arc in between any possible pair of edges with two possible orientations and changes the spin of the two corresponding adjacent segments by $\pm 1 / 2$. Therefore it adds two more vertices. Working at the gauge variant level (there are more gauge variant SNWFs than invariant ones) this also changes the magnetic quantum numbers at the end points of all three edges by $\pm 1 / 2$ which results in an additional factor of $4^{3}$ at most. Hence per vertex of valence $n(v)$ we get in this way no more than $4 \cdot 2 \cdot 4^{3} n(v)(n(v)-1) / 2=4^{4} n(v)(n(v)-1)$ new spin network states from the first term.

Second term. The second term is the square of the first term as far as the counting of new states is concerned. Hence we get $4^{8} n(v)^{2}(n(v)-1)^{2}$ new spin network states from the second term depending on two more arcs and four more vertices.

Now in order that any of those is diffeomorphic to $T_{s_{1}}$ the graph $\gamma\left(s_{0}([s])\right)$ must have one or two edges less than $\gamma\left(s_{1}\right)$ and two or four vertices less than $\gamma\left(s_{1}\right)$. Moreover, the spins of the segments of edges adjacent to the arcs must differ by $\pm 1 / 2$ and the magnetic quantum numbers of arcs and edges must differ by $\pm 1 / 2$. We conclude that if $N_{1}$ is the maximal valence of a vertex of $\gamma\left(s_{1}\right)$ then the number of [ $s$ ] that can contribute is bounded by $4^{8} N_{1}^{4} \mid V\left(\gamma\left(s_{1}\right) \mid\right.$ which depends only on $\left[s_{1}\right]$. The same applies to $s_{2}$ of course. The actually contributing number of $[s]$ is certainly smaller than the maximum of $4^{8} N_{1}^{4} \mid V\left(\gamma\left(s_{1}\right)\left|, 4^{8} N_{2}^{4}\right| V\left(\gamma\left(s_{2}\right) \mid\right.\right.$.

(2) Let us now fix $\left[s_{2}\right]$ and let $\left[s_{1}\right]$ run. There are only $4^{8} N_{2}^{4} \mid V\left(\gamma\left(s_{2}\right) \mid\right.$ classes $[s]$ which can contribute no matter which $\left[s_{1}\right]$ we choose. By a similar argument, for each of those $[s]$ the number of $\left[s_{1}\right]$ which lead to a non-vanishing contribution is bounded by $4^{8} N^{4} \mid V\left(\gamma\left(s_{0}([s])\right) \mid+2\right.$ where $N$ is the maximal vertex valence of $\gamma\left(s_{0}([s])\right)$. Since $N=N_{2}$ and $\left|V\left(\gamma\left(s_{0}([s])\right)\right)\right| \leqslant\left|V\left(\gamma\left(s_{2}\right)\right)\right|$ we conclude that $Q_{\mathbf{M}}\left(T_{\left[s_{1}\right]}, T_{\left[s_{2}\right]}\right)$ is non-vanishing for at $\operatorname{most} 4{ }^{16} N_{2}^{8}\left|V\left(\gamma\left(s_{0}\left(\left[s_{2}\right]\right)\right)\right)\right|^{2}$ of the classes $\left[s_{1}\right]$.

We thus have shown that there is a positive symmetric operator $\widehat{M}$ with dense domain $\mathcal{D}_{\text {diff }}$, the finite linear span of the $T_{[s]}$, defined by (3.2) whose quadratic form coincides with $Q_{\mathrm{M}}$ on the form domain $D\left(Q_{\mathrm{M}}\right)=\mathcal{D}_{\text {diff }}$. Hence by theorem 3.1 (iii) $Q_{\mathrm{M}}$ has a positive closure and induces a unique self-adjoint (Friedrichs) extension of $\widehat{\mathbf{M}}$ by theorem 3.1 which we denote by $\widehat{\mathbf{M}}$ as well.

(ii) Note that the construction of the solutions of $\hat{H}^{\prime}(N) l=0$ for all $N$ (which produces zero eigenvectors, i.e. normalizable elements of $\mathcal{H}_{\text {diff }}$ ) displayed explicitly in [13] can be directly transcribed to the construction of solutions to $\widehat{\mathbf{M}} l=0$. Namely, $\widehat{\mathbf{M}} l=0$ implies $Q_{\mathrm{M}}(l, l)=0$ which in turn enforces $l\left(\hat{C}_{v}^{\dagger} T_{s_{0}([s])}\right)=0$ for all $[s]$ and all $v \in V\left(\gamma\left(s_{0}([s])\right)\right.$. This is equivalent to $l\left(\hat{C}^{\dagger}(N) T_{s}\right)=0$ for all $s$ and all $N$ where $\hat{C}^{\dagger}(N)$ is defined identically as $\hat{H}^{\dagger}(N)$ just that one of the volume operators is replaced by two times its square root. Thus, in particular $T_{[s]}$ where $s$ has no extraordinary edges [13] are normalizable solutions. 
Hence the master constraint operator has a point kernel at least as rich as the Hamiltonian constraint. Moreover, it gives us additional flexibility in the following sense: in order to have a consistent constraint algebra the action of the Hamiltonian constraint had to be trivial at the vertices that it creates itself. However, the master constraint does not have to satisfy any non-trivial constraint algebra, hence this restriction can be relaxed to be less local. Whether such modifications lead to a sufficiently large semiclassical sector is, of course, not clear a priori and is subject to a detailed semiclassical analysis [21].

We close this section with a remark on matter coupling and the extended master constraint:

(1) Matter coupling. The derivation and proof of closure of the quadratic form of the master constraint remains the same for all known matter [16] because the essential part of the derivation and proof respectively was that the attachment of the loop (arc) to a given graph follows diffeomorphism covariant rules. This was done universally for matter and geometry in [16].

(2) Extended master constraint. In contrast to the master constraint considered in the previous two sections the extended master constraint also involves the spatial diffeomorphism constraint (or even the Gauss constraint). Its classical expression is given by [22]

$\mathbf{M}_{E}=\int_{\sigma} \mathrm{d}^{3} x \frac{H^{2}+q^{a b} H_{a} H_{b}}{\sqrt{\operatorname{det}(q)}}, \quad \mathbf{M}_{E E}=\int_{\sigma} \mathrm{d}^{3} x \frac{H^{2}+q^{a b} H_{a} H_{b}+H_{j} H_{j}}{\sqrt{\operatorname{det}(q)}}$

where $H, H_{a}, H_{j}$ denote Hamiltonian, spatial diffeomorphism and Gauss constraint, respectively. Both constraints are spatially diffeomorphism invariant. However, $\mathbf{M}_{E}$ allows us to implement both the Hamiltonian and the spatial diffeomorphism constraint on $\mathcal{H}_{\text {kin }}$ (and $\mathbf{M}_{E E}$ also the Gauss constraint in addition) provided we implement the corresponding operators in a non-graph changing fashion. In [22] we showed how to do that using the notion of a minimal loop which is a loop (average) within the graph on which the constraint acts. It follows that instead of using dual operators we can directly work with operators on $\mathcal{H}_{\text {kin }}$ and their adjoints so that the construction of the quadratic form can be sidestepped.

\section{Physical inner product and Dirac observables}

Given the self-adjoint master constraint operator $\widehat{\mathbf{M}}$ of the previous section one would now like to use the machinery of the direct integral decomposition reviewed in [23] in order to define the physical Hilbert space. However, there is one additional obstacle: while the spectral theorem holds also in non-separable Hilbert spaces, the direct integral decomposition can be performed only in the separable case. However, $\mathcal{H}_{\text {diff }}$ is not separable unless, possibly, if we admit semianalytic homeomorphisms which remove the continuous moduli [27] for vertices of valence five or higher. Now using homeomorphisms is forbidden because we must use the volume operator [20] rather than [19] as shown in [28, 29] which depends on a $C^{(1)}$ structure and which is absolutely crucial in order that $\widehat{\mathbf{M}}$ or $\hat{H}^{\prime}(N)$ be even densely defined. Thus, the direct integral method seems not to be applicable.

Fortunately, $\mathcal{H}_{\text {diff }}$ can be decomposed as an uncountably infinite direct sum of separable Hilbert spaces as follows [22]:

Definition 4.1. We say that two embedded graphs $\gamma_{1}, \gamma_{2}$ are $\theta$-equivalent (or homotopic up to the degeneracy type) provided that that there exists a semianalytic diffeomorphism $\varphi \in$ Diffo $f_{\text {sa }}(\sigma)$ such that

(1) $V\left(\varphi\left(\gamma_{1}\right)\right)=V\left(\gamma_{2}\right)$. 
(2) $\varphi\left(\gamma_{1}\right), \gamma_{2}$ are topologically equivalent, that is, all vertices have the same connectivities with other vertices and edges are braided (knotted) and oriented the same way. Denote by $b: E\left(\varphi\left(\gamma_{1}\right)\right) \rightarrow E\left(\gamma_{2}\right)$ the corresponding bijection.

(3) At each $v \in V\left(\gamma_{2}\right)$ and for each triple $e_{1}, e_{2}, e_{3} \in E\left(\varphi\left(\gamma_{1}\right)\right)$ of distinct edges the corresponding sign functions coincide, that is, $\epsilon\left(e_{1}, e_{2}, e_{3}\right)=\epsilon\left(b\left(e_{1}\right), b\left(e_{2}\right), b\left(e_{3}\right)\right)$, where $\epsilon\left(e_{1}, e_{2}, e_{3}\right)$ equals $+1,-1,0$ respectively if the tangents of the edges on their common starting point are right oriented, left oriented or co-planar respectively.

Denote by $[\Gamma]$ the set of diffeomorphism equivalence classes $[\gamma]$ of graphs $\gamma \in \Gamma$ and by $(\Gamma)$ the set of $\theta$-equivalence classes $(\gamma)$ of graphs. Given $(\gamma)$, let $\Theta_{(\gamma)}^{\prime}$ be the set of moduli that are necessary to specify all the $\left[\gamma^{\prime}\right]$ with $\left(\gamma^{\prime}\right)=(\gamma)$. Hence any element $[\gamma] \in[\Gamma]$ is now uniquely specified by a pair $\left((\gamma), \theta^{\prime}\right) \in(\Gamma) \times \Theta_{(\gamma)}^{\prime}$. Let

$$
\Theta^{\prime}:=\times_{(\gamma) \in(\Gamma)} \Theta_{(\gamma)}^{\prime} \ni \theta=\left\{\theta_{(\gamma)}^{\prime}\right\}_{(\gamma) \in(\Gamma)} .
$$

Then the direct sum of Hilbert spaces

$$
\mathcal{H}_{\text {diff }}=\oplus_{[\gamma] \in[\Gamma]} \mathcal{H}_{\text {diff }}^{[\gamma]}
$$

where $\mathcal{H}_{\text {diff }}^{[\gamma]}$ is the closure of the finite linear span of $T_{[s]}$ with non-trivial representations on all edges can be decomposed also as

$$
\mathcal{H}_{\text {diff }}=\mathcal{H}_{\text {diff }}^{0} \oplus \oplus_{\theta^{\prime} \in \Theta^{\prime}} \oplus_{(\gamma) \in(\Gamma)-(\Gamma)_{0}} \mathcal{H}_{\text {diff }}^{\left((\gamma), \theta_{(\gamma)}^{\prime}\right)}=: \bigoplus_{\theta^{\prime} \in \Theta^{\prime}} \mathcal{H}_{\text {diff }}^{\prime \theta^{\prime}}
$$

where $\mathcal{H}_{\text {diff }}^{0}=\bigoplus_{[\gamma] \in[\Gamma]_{0}} \mathcal{H}_{[\gamma]}$ and $(\Gamma)_{0}=[\Gamma]_{0}$ is the subset of graphs without moduli. We claim that all the $\mathcal{H}_{\text {diff }}^{\prime \prime^{\prime}}$ are separable and mutually unitarily equivalent. Unitary equivalence is clear, we just have to map the corresponding points $\theta^{\prime}$. Separability follows from the fact that at fixed $\theta$ a spin network label equivalence class is completely specified (1) by the number of vertices and their connectivities, (2) by the braiding and orientation of the corresponding edges and (3) by the spin and intertwining quantum numbers. Each of the three label sets is countable, hence it has the cardinality of $\mathbb{N}^{3}$ which is countable.

Unfortunately, the sectors $\mathcal{H}_{\text {diff }}^{\prime \theta^{\prime}}$ are generically not left invariant by $\widehat{\mathbf{M}}$ : this follows from the fact that $\widehat{\mathbf{M}}$ can have non-vanishing matrix elements between $T_{[s]}, T_{\left[s^{\prime}\right]}$ where $\gamma(s), \gamma\left(s^{\prime}\right)$ differ by an arc. Now $(\gamma(s)),\left(\gamma\left(s^{\prime}\right)\right)$ have the same moduli space, $\Theta_{(\gamma(s))}^{\prime}=\Theta_{\left(\gamma\left(s^{\prime}\right)\right)}^{\prime}$, because the two three-valent vertices created by the arc do not require additional moduli information and the $\left[s^{\prime}\right]$ obtained from $[s]$ is such that the moduli coincide. However, in the Hilbert space $\mathcal{H}_{\text {diff }}^{\prime \theta}$ the moduli assigned to $\left(\gamma\left(s^{\prime}\right)\right)$ might be different from those assigned to $(\gamma(s))$. Hence the $\theta$ sectors described above are not preserved. We could, of course, identify by hand these $\theta$-sectors and make $\mathcal{H}_{\text {diff }}$ separable altogether. The motivation for doing that is that every $\theta$-sector presumably already contains the physically relevant information encoded by $(\Gamma)$. But this is not what the formalism forces us to do.

It is therefore safer to do something else: we can combine the $\theta$-moduli classification with the classification by sources $\mathcal{S}_{0}$ and derived spin nets $\mathcal{S}_{n}\left(s_{0}\right)$ of level $n$ developed in [13] as follows: denote by $\left[\mathcal{S}_{0}\right]$ the set of diffeomorphism equivalence classes of sources. For any two representatives $s_{1}\left(\left[s_{0}\right]\right), s_{2}\left(\left[s_{0}\right]\right) \in \mathcal{S}_{0}$ the set of diffeomorphism equivalence classes of the members of the derived spin nets of level $n$ of $\mathcal{S}_{n}\left(s_{1}\left(\left[s_{0}\right]\right)\right), \mathcal{S}_{n}\left(s_{2}\left(\left[s_{0}\right]\right)\right)$ coincide, i.e. they depend only on $\left[s_{0}\right]$. We will denote this set therefore by $\left[\mathcal{S}_{n}\right]\left(\left[s_{0}\right]\right)$. We note that the moduli parameters of all the $[s] \in\left[\mathcal{S}_{n}\right]\left(\left[s_{0}\right]\right), n=0,1, \ldots$ are completely determined by those of $\left[s_{0}\right]$. The completion of the finite linear span of these $T_{[s]}$ will be denoted by $\mathcal{H}_{\text {Diff }}^{\left[s_{0}\right]}$ and this Hilbert space is separable by construction. Now the following issue arises: the action of $\widehat{\mathbf{M}}$ consists in adding and removing arcs to a graph and sometimes it reduces the valence of a vertex by one or two units. It therefore happens that given $\left[s_{0}\right] \neq\left[s_{0}^{\prime}\right]$ with $\left(s_{0}\right)=\left(s_{0}^{\prime}\right)$ that the 
set $\left[\mathcal{S}_{n}\right]\left(\left[s_{0}\right]\right) \cap\left[\mathcal{S}_{n}\right]\left(\left[s_{0}^{\prime}\right]\right)$ is not empty. For instance a five-valent vertex, which has moduli, could be turned into a three-valent one which does not have moduli. Hence it is almost but not quite true that $\mathcal{H}_{\text {diff }}$ is the uncountable direct sum of the $\mathcal{H}_{\text {diff }}^{\left[s_{0}\right]},\left[s_{0}\right] \in\left[\mathcal{S}_{0}\right]$.

Let us write $\left[s_{0}\right]=\left(\left(s_{0}\right), \theta_{\left(s_{0}\right)}:=\theta_{\left(\gamma\left(s_{0}\right)\right)}\right)$ where $\left(s_{0}\right)$ is the $\theta$-equivalence class of $s_{0}$ which is determined by the $\left(\gamma\left(s_{0}\right)\right)$. Let $\left(\mathcal{S}_{0}\right)$ be the set of those $\left(s_{0}\right)$ and let $\Theta^{\prime}$ be the collection of the $\theta_{\left(s_{0}\right)},\left(s_{0}\right) \in\left(\mathcal{S}_{0}\right)$. Then

$$
\mathcal{H}_{\text {diff }}=\cup_{\theta \in \Theta} \mathcal{H}_{\text {diff }}^{\theta}:=\cup_{\left(s_{0}\right) \in\left(\mathcal{S}_{0}\right)} \mathcal{H}_{\text {diff }}^{\left(\left(s_{0}\right), \theta_{\left(s_{0}\right)}\right)} .
$$

Note that the unions are almost direct sums but not quite as just pointed out. However, each of the spaces $\mathcal{H}_{\text {Diff }}^{\theta}$ is a separable and $\widehat{\mathbf{M}}$-invariant subspace of $\mathcal{H}_{\text {Diff }}$ and all of them are mutually isomorphic. Moreover, each of them contains information about all $\theta$-equivalence classes of spin network states and therefore all the physically relevant information.

Thus, while these are not sectors in the strict sense, we may just pick one of these subspaces and apply the direct integral decomposition method to it.

Theorem 4.1. There is a unitary operator $V$ such that $V \mathcal{H}_{\mathrm{diff}}^{\theta}$ is the direct integral Hilbert space

$$
\mathcal{H}_{\text {diff }}^{\theta} \propto \int_{\mathbb{R}^{+}}^{\oplus} \mathrm{d} \mu(\lambda) \mathcal{H}_{\text {diff }}^{\theta}(\lambda)
$$

where the measure class of $\mu$ and the Hilbert spaces $\mathcal{H}_{\text {Diff }}^{\theta}(\lambda)$, in which $V \widehat{\mathbf{M}} V^{-1}$ acts by multiplication by $\lambda$, are $\mu$-uniquely determined. The physical Hilbert space is given by ${ }^{3}$ $\mathcal{H}_{\text {phys }}^{\theta}=\mathcal{H}_{\text {diff }}^{\theta}(0)$.

Dirac observables could now be constructed from spatially diffeomorphism invariant operators which preserve any $\mathcal{H}_{\text {diff }}^{\theta}$, e.g. by using the ergodic projection technique of [22] or by the partial observable technique of [30]. Any spatially diffeomorphism invariant operator regularized in the same fashion as the Hamiltonian constraint operator has the property to preserve each of the subspaces $\mathcal{H}_{\text {diff }}^{\theta}$ separately, hence this is no restriction.

\section{The classical master equation for selecting weak Dirac observables}

In the case of boundaries of $\sigma$, the classical Hamiltonian constraint has to be supplemented by boundary terms in order to be functionally differentiable (i.e. its Hamiltonian vector field is well defined) for lapse functions which do not vanish on the boundaries. At first sight, the master constraint needs to be twice functionally differentiable in order that the master equation definition of weak Dirac observables $\{O,\{O, \mathbf{M}\}\}_{\mathbf{M}=0}=0$ [22] makes sense and since there are no smearing (lapse) functions involved the issue of boundary terms could be non-trivial. We will now show that the master constraint is actually more regular than the Hamiltonian constraint as far as the master equation is concerned and the issue of boundary terms does not arise in the case of asymptotic flatness (no interior boundaries). We will just sketch this for the piece $H_{E}(x)$ and for pure gravity, similar remarks hold for the full Lorentzian constraint and matter coupling.

Let us write $\mathbf{M}=\int \mathrm{d}^{3} x C(x)^{2}, C(x)^{2}=H_{E}(x)^{2} / \sqrt{\operatorname{det}(q)}(x)$. Recall [31] that the boundary conditions at spatial infinity are such that the components of $A$ fall off as $r^{-2}$ while the components of $E-E_{0}$ fall off as $r^{-1}$ where $E_{0}$ is the fixed boundary value of $E$ compatible with the Euclidean metric. Here $r$ is with respect to an asymptotically Cartesian system of

3 The spaces $\mathcal{H}_{\text {diff }}^{\theta}(\lambda)$ are defined up to measure $\mu$ zero sets. See [23] for physical criteria to choose an appropriate candidate. 
coordinates and with respect to asymptotic reflections $A$ and $E-E_{0}$ respectively have to be odd and even respectively. It follows that the tangent vectors $\delta A, \delta E$ fall off as $A, E-E_{0}$ and have the same reflection properties.

We conclude from the definition of $H_{E}$ in (2.1) that $C(x)$ falls off as $r^{-3}$ so that the integral of the master constraint itself converges. In what follows we will symbolically write $\mathrm{d} f, \mathrm{D} f$ for a partial or $S U(2)$ covariant derivative of a function of $f$ which will be enough to determine the fall-off properties of various terms. We will also write $Q$ for a generic function of $E$ alone which asymptotically converges to a constant. We assume of course that $O$ itself is functionally differentiable, that is

$$
\delta O=\int_{\sigma}[I \cdot \delta A+J \cdot \delta E]
$$

converges, so that $I$ falls off at least as $r^{-1}$ with even parity and $J$ as $r^{-2}$ with odd parity. The variation of $\mathbf{M}$ itself gives

$$
\delta \mathbf{M}=\int_{\sigma}\left[\left(\mathrm{D}\left(Q_{1} C\right)\right) \cdot \delta A+\left(Q_{2} C\right) \cdot \delta E\right] .
$$

The first term involves an integration by parts but since $C$ falls off as $r^{-3}$ no boundary term is picked up.

Combining (5.1) and (5.2) we find for the first-order Poisson bracket

$$
\{O, \mathbf{M}\}=\int_{\sigma}\left[I \cdot\left(Q_{2} C\right)-J \cdot \mathrm{D}\left(Q_{1} C\right)\right] .
$$

The integrand falls off at least as $r^{-4}$ and thus converges. Varying (5.3) again at $\mathbf{M}=0$ we just need to consider the terms that result from variations of $C$ (otherwise, that is when considering variations off the constraint surface $\mathbf{M}=0$, we must make suitable assumptions about the variations of $I, J)$. Hence

$\delta\{O, \mathbf{M}\}_{\mathbf{M}=0}=\int_{\sigma}\left[I \cdot\left(Q_{2} \delta C\right)-J \cdot \mathrm{D}\left(Q_{1} \delta C\right)\right]=\int_{\sigma}\left[I Q_{2}+(\mathrm{D} J) Q_{1}\right] \cdot \delta C$

In the second step we had to perform an integration by parts in the second term. Since $\delta C$ falls off at least as $r^{-3}$ while $J$ falls off at least as $r^{-2}$ no boundary term is picked up. Performing a further integration by parts we can finish (5.4) with the result

$\delta\{O, \mathbf{M}\}_{\mathbf{M}=0}=\int_{\sigma}\left[\left(\mathrm{D}\left(Q_{1}\left(I Q_{2}+(\mathrm{D} J) Q_{1}\right)\right)\right) \cdot \delta A+\left(F Q_{4}\right)\left(I Q_{2}+(\mathrm{D} J) Q_{1}\right) \cdot \delta E\right]$

where as before $F$ denotes the curvature of $A$. We have dropped a term proportional to $C$ in the variation of $E$. The integral (5.5) evidently converges because the integrand falls off at least as $r^{-4}$.

Combining (5.1) and (5.5) we find

$\left.\{O,\{O, \mathbf{M}\}\}_{\mathbf{M}=0}=\int_{\sigma}\left[I \cdot\left(F Q_{4}\right)\left(I Q_{2}+(\mathrm{D} J) Q_{1}\right)\right]-J \cdot\left(\mathrm{D}\left(Q_{1}\left(I Q_{2}+(\mathrm{D} J) Q_{1}\right)\right)\right)\right]$

and the integral still converges since the integrand falls off as $r^{-4}$ at least. Note that the parity properties never had to be used.

We conclude that the master equation is well defined without additional boundary terms for the master constraint with asymptotically flat boundary conditions at least on the constraint surface $\mathbf{M}=0$ for once functionally differentiable $O$ and off the constraint surface for suitable twice differentiable $O$. 


\section{Conclusions}

The results of [22] and this paper establish that a self-adjoint, positive master constraint operator for LQG exists. The results of [23] show that the existence of a physical inner product by direct integral methods is automatic. The results of [24] demonstrate that the master constraint programme leads to the expected physical results in a large number of rather generic model situations, e.g., in examples with second class constraints, with structure functions, an infinite number of degrees of freedom etc.

Taken together, the master constraint programme has good chances to overcome the difficulties that have hindered progress with the Hamiltonian constraint over the past decade. The next step is to check whether the master constraint has the correct classical limit and to develop approximation methods that enable us to construct physical states and the physical inner product explicitly.

\section{Acknowledgments}

We would like to thank Yongge Ma and Muxin Han, who have arrived at similar results from a different perspective, for discussions. It is a pleasure to thank Yongge Ma for his generous hospitality during the author's visit to Beijing Normal University. This research project has been supported in part by funds from NSERC of Canada to the Perimeter Institute for Theoretical Physics.

\section{References}

[1] Hojman S A, Kuchar K and Teitelboim C 1976 Geometrodynamics regained Ann. Phys., NY 96 88-135

[2] Rovelli C 2004 Quantum Gravity (Cambridge: Cambridge University Press)

Thiemann T 2006 Modern Canonical Quantum General Relativity (Cambridge: Cambridge University Press) (draft available as Preprint gr-qc/0110034, submitted)

[3] Rovelli C 1998 Loop quantum gravity Living Rev. Rel. 11 (Preprint gr-qc/9710008)

Thiemann T 2003 Lectures on loop quantum gravity Lect. Notes Phys. 631 41-135 (Preprint gr-qc/0210094)

Ashtekar A and Lewandowski J 2004 Background independent quantum gravity: a status report Class. Quantum Grav. 21 R53 (Preprint gr-qc/0404018)

Smolin L 2004 An invitation to loop quantum gravity Preprint hep-th/0408048

[4] Ashtekar A 1987 Phys. Rev. D 361587

Barbero F 1995 Phys. Rev. D 515507

Barbero F 1995 Phys. Rev. D 515498

[5] Gambini R and Trias A 1980 Phys. Rev. D 221380

Giles R 1981 Phys. Rev. D 82160

Di Bartolo C, Nori F, Gambini R and Trias A 1983 Lett. Nuovo Cimento 38497

Gambini R and Trias A 1986 Nucl. Phys. B 278436

Jacobson T and Smolin L 1988 Nonperturbative quantum geometries Nucl. Phys. B 299295

Rovelli C and Smolin L 1990 Loop space representation of quantum general relativity Nucl. Phys. B 33180

[6] Sahlmann H 2002 When do measures on the space of connections support the triad operators of loop quantum gravity Preprint gr-qc/0207112

Sahlmann H 2002 Some comments on the representation theory of the algebra underlying loop quantum gravity Preprint gr-qc/0207111

Okolow A and Lewandowski J 2003 Diffeomorphism covariant representations of the holonomy flux algebra Class. Quantum Grav. 20 3543-68 (Preprint gr-qc/03027112)

Okolow A and Lewandowski J 2005 Automorphism covariant representations of the holonomy flux *-algebra Class. Quantum Grav. 22 657-80 (Preprint gr-qc/0405119)

Sahlmann H and Thiemann T 2003 On the superselection theory of the Weyl algebra for diffeomorphism invariant quantum gauge theories Preprint gr-qc/0302090

Sahlmann H and Thiemann T 2003 Irreducibility of the Ashtekar-Isham-Lewandowski representation Preprint gr-qc/0303074 
Lewandowski J, Okolow A, Sahlmann H and Thiemann T 2005 Uniqueness of diffeomorphism invariant states on holonomy-flux algebras Preprint gr-qc/0504147

Fleischhack C 2004 Representations of the Weyl algebra in quantum geometry Preprint math-ph/0407006

[7] Bratteli O and Robinson D W 1997 Operator Algebras and Quantum Statistical Mechanics vols 1 and 2 (Berlin: Springer)

[8] Ashtekar A and Isham C J 1992 Representations of the holonomy algebras of gravity and non-Abelian gauge theories Class. Quantum Grav. 91433 (Preprint hep-th/9202053)

Ashtekar A and Lewandowski J 1994 Representation theory of analytic holonomy $C^{\star}$ algebras Knots and Quantum Gravity ed J Baez (Oxford: Oxford University Press)

[9] Reed M and Simon B 1980 Methods of Modern Mathematical Physics vols 1-4 (Boston, MA: Academic)

[10] Ashtekar A, Lewandowski J, Marolf D, Mourão J and Thiemann T 1995 Quantization of diffeomorphism invariant theories of connections with local degrees of freedom J. Math. Phys. 36 6456-93 (Preprint gr-qc/9504018)

[11] Thiemann T 1996 Anomaly-free formulation of non-perturbative, four-dimensional Lorentzian quantum gravity Phys. Lett. B 380 257-64 (Preprint gr-qc/9606088)

[12] Thiemann T 1998 Quantum spin dynamics (QSD) Class. Quantum Grav. 15 839-73 (Preprint gr-qc/9606089)

[13] Thiemann T 1998 Quantum spin dynamics (QSD): II. The kernel of the Wheeler-deWitt constraint operator Class. Quantum Grav. 15 875-905 (Preprint gr-qc/9606090)

[14] Thiemann T 1998 Quantum spin dynamics (QSD): III. Quantum constraint algebra and physical scalar product in quantum general relativity Class. Quantum Grav. 15 1207-47 (Preprint gr-qc/9705017)

[15] Thiemann T 1998 Quantum spin dynamics (QSD): IV. 2+1 Euclidean quantum gravity as a model to test 3+1 Lorentzian quantum gravity Class. Quantum Grav. 15 1249-80 (Preprint gr-qc/9705018)

[16] Thiemann T 1998 Quantum spin dynamics (QSD): V. Quantum gravity as the natural regulator of the Hamiltonian constraint of matter quantum field theories Class. Quantum Grav. 15 1281-314 (Preprint gr-qc/9705019)

[17] Thiemann T 1998 Quantum spin dynamics (QSD): VI. Quantum Poincaré algebra and a quantum positivity of energy theorem for canonical quantum gravity Class. Quantum Grav. 15 1463-85 (Preprint gr-qc/9705020)

[18] Thiemann T 1998 Kinematical Hilbert spaces for fermionic and Higgs quantum field theories Class. Quantum Grav. 15 1487-512 (Preprint gr-qc/9705021)

[19] Rovelli C and Smolin L 1995 Discreteness of volume and area in quantum gravity Nucl. Phys. B 442593 Rovelli C and Smolin L 1995 Nucl. Phys. B 456734 (erratum)

[20] Ashtekar A and Lewandowski J 1997 Quantum theory of geometry: II. Volume operators Adv. Theor. Math. Phys. 1 388-429

[21] Thiemann T 2001 Quantum spin dynamics (QSD): VII. Symplectic structures and continuum lattice formulations of gauge field theories Class. Quantum Grav. 18 3293-338 (Preprint hep-th/0005232)

Thiemann T 2001 Gauge field theory coherent states (GCS): I. General properties Class. Quantum Grav. 18 2025-64 (Preprint hep-th/0005233)

Thiemann T and Winkler O 2001 Gauge field theory coherent states (GCS): II. Peakedness properties Class. Quantum Grav. 18 2561-636 (Preprint hep-th/0005237)

Thiemann T and Winkler O 2001 Gauge field theory coherent states (GCS): III. Ehrenfest theorems Class. Quantum Grav. 18 4629-81 (Preprint hep-th/0005234)

Thiemann T and Winkler O 2001 Gauge field theory coherent states (GCS): IV. Infinite tensor product and thermodynamic limit Class. Quantum Grav. 18 4997-5033 (Preprint hep-th/0005235)

Sahlmann H, Thiemann T and Winkler O 2001 Coherent states for canonical quantum general relativity and the infinite tensor product extension Nucl. Phys. B 606 401-40 (Preprint gr-qc/0102038)

Thiemann T 2006 Complexifier coherent states for canonical quantum general relativity Class. Quantum Grav. 23 2063-117 (Preprint gr-qc/0206037)

[22] Thiemann T 2006 The phoenix project: master constraint programme for loop quantum gravity Class. Quantum Grav. 23 2211-47 (Preprint gr-qc/0305080)

[23] Dittrich B and Thiemann T 2006 Testing the master constraint programme for loop quantum gravity: I. General framework Class. Quantum Grav. 23 1025-65 (Preprint gr-qc/0411138)

[24] Dittrich B and Thiemann T 2006 Testing the master constraint programme for loop quantum gravity: II. Finite-dimensional systems Class. Quantum Grav. 23 1067-88 (Preprint gr-qc/0411139)

Dittrich B and Thiemann T 2006 Testing the master constraint programme for loop quantum gravity: III. SL $(2 R)$ models Class. Quantum Grav. 23 1089-120 (Preprint gr-qc/0411140)

Dittrich B and Thiemann T 2006 Testing the master constraint programme for loop quantum gravity: IV. Free field theories Class. Quantum Grav. 23 1121-42 (Preprint gr-qc/0411141)

Dittrich B and Thiemann T 2006 Testing the master constraint programme for loop quantum gravity: V. Interacting field theories Class. Quantum Grav. 23 1143-62 (Preprint gr-qc/0411142) 
[25] Immirzi G 1997 Quantum gravity and Regge calculus Nucl. Phys. Proc. Suppl. 5765 (Preprint gr-qc/9701052) Rovelli C and Thiemann T 1998 Phys. Rev. D 57 1009-14

[26] Potter M D 1990 Mengentheorie (Berlin: Spektrum Akademischer) chapter 5

Cameron P J 1999 Sets, Logic and Categories (Berlin: Springer)

[27] Grot N and Rovelli C 1996 Moduli space structure of knots with intersections J. Math. Phys. 37 3014-21 (Preprint gr-qc/9604010)

Fairbairn W and Rovelli C 2004 Separable Hilbert space in loop quantum gravity J. Math. Phys. 45 2802-14 (Preprint gr-qc/0403047)

[28] Giesel K and Thiemann T 2005 Consistency check on volume and triad operator quantisation in loop quantum gravity: I Preprint gr-qc/0507036

[29] Giesel K and Thiemann T 2005 Consistency check on volume and triad operator quantisation in loop quantum gravity: II Preprint gr-qc/0507037

[30] Dittrich B 2004 Partial and complete observables for Hamiltonian constrained systems Preprint gr-qc/0411013 Dittrich B 2005 Partial and complete observables for canonical general relativity Preprint gr-qc/0507106 Thiemann T 2004 Reduced phase space quantization and Dirac observables Preprint gr-qc/0411031

[31] Thiemann T 1995 Generalized boundary conditions for general relativity for the asymptotically flat case in terms of ashtekar's variables Class. Quantum Grav. 12 181-98 (Preprint gr-qc/9910008) 